\title{
Radio frequency power sensor based on MEMS technology
}

\author{
Luis J. Fernandez, Eelke Visser, Javier Sese, Remco Wiegerink, Jaap Flokstra, Henri \\ Jansen, Miko Elwenspoek
}

MESA+ Institute for Nanotechnology, University of Twente, P.O. Box 217, 7500AE, Enschede, The Netherlands

l.j.fernandez@el.utwente.nl

\begin{abstract}
We present the first measurement results of a power sensor for radio frequency (rf) signals $(50 \mathrm{kHz}-40 \mathrm{GHz})$ with almost no dissipation during the measurement. This sensor is, therefore, a 'through' power sensor, that means that the $r f$ signal is available during the measurement of its power. The power detection has been realized by measuring capacitively the movement of a grounded aluminum membrane, which is suspended above the transmission line carrying the rf signal. The power sensor is thus a capacitive MEMS technology based sensor. The fabrication is done by aluminum surface micromachining on an AF45 glass wafer. We measured the capacitance as a function of the applied $r$ power and found a linear relationship as predicted from theory.
\end{abstract}

\section{Keywords}

Power sensor, radio frequency, capacitive MEMS.

\section{INTRODUCTION}

The existing technology for power detection for radio frequency (rf) signals is based on thermistors, thermocouples and diodes. These are terminating devices, i.e. the signal is dissipated during the power measurement. A completely different philosophy to realize power detection has been recently proposed, in a way that the signal is available during the measurement [1-3]. The power measurement is based on the movement detection of a grounded membrane suspended above a planar transmission line where the signal is traveling. This movement is measured capacitively. The first part of this paper explains the operation principle of the sensor. Then, the design and fabrication are shown. Finally the experiments on first prototypes are presented and compared to theory. As a conclusion, we have developed the first "through" sensor for if signals that can be inserted in an rf circuit without dissipating (part of) the signal. The fabrication is CMOS-compatible and allows easy cohabitation with microelectronics.

\section{OPERATION PRINCIPLE}

An $\mathrm{rf}$ signal that is transported on a transmission line with characteristic impedance $Z_{0}$ has a power level that is given by $V_{r m s}^{2} / Z_{0}$, where $V_{r m s}$ is the rms voltage of the rf signal. When a capacitor is connected in parallel with the transmission line, an electrical force will appear between the capacitor plates: $F=C(V \cos \omega t)^{2} /(2 d)$ where $C$ is the capacitance and $\mathrm{d}$ is the distance between the capacitor plates. One of the plates is movable supported with spring constant $\mathrm{k}$. For frequencies of the signal that are much higher than the mechanical resonance frequency of the movable plate, the plate is only sensitive to the rms value of the force, that is proportional to the power level: $F=C V_{r m s}^{2} /(2 d)$. Therefore, the power level can be deduced by detecting the movement of the plate. In practice the movement is detected as a capacitance change between a measuring electrode and the movable plate.

The addition of the capacitor will change the impedance of the transmission line and hence reflection losses are introduced. The reflection parameter is given by $S_{I I}=10 \cdot \log \left(|\Gamma|^{2}\right)$ where $\Gamma=\left(Z-Z_{0}\right) /\left(Z+Z_{0}\right)$ and $Z=Z_{0} \quad / /$ $1 / j \omega C$ as we can see from the schematic of the circuit in figure 1 . The transmission parameter, $|T|^{2}=1-|\Gamma|^{2}$, assuming no conduction and dielectric losses, is given by $S_{12}=10 \cdot \log \left(|T|^{2}\right)$. The minimum power that can be detected is primarily limited by the measuring electronics and ultimately by the thermo-mechanical noise of the membrane.

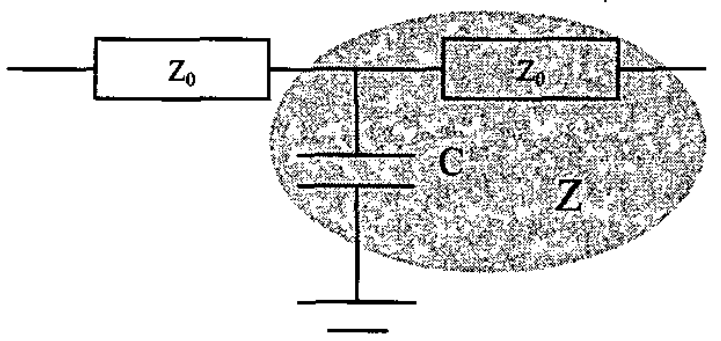

Figure 1. Schematic of the circult.

\section{DESIGN}

In order to get the best response of the sensor for rf signals, two different parts of the sensor have to be studied in detail. The first one is the electrical study of the transmission line where the signal is traveling through, and the other is the mechanical design of the sensing capacitor needed for the power measurements.

\section{Transmission line}

We decided to use Coplanar Waveguides (CPW), see figure 2 , due to its large frequency range and because this allows planar processing, which is perfectly adapted to the micro system technology. The CPW line consists of 3 metal lines on top of a dielectric material. The lines at the sides are grounded and the central one is carrying the rf signal. The impedance of the line is defined by geometrical and material characteristics, such as the distance between the signal line and the grounded lines, and the dielectric constant of the dielectric material used as a support. The CPW is de- 
signed to have $50 \mathrm{Ohm}$ characteristic impedance. In order to design such a $\mathrm{CPW}$, simulation were done using FEMLAB [4], where capacitance calculations were performed in order to estimate the closest dimensions to the 50 Ohm configuration. The materials chosen for the fabrication were aluminum due to its high conductivity and easy deposition for the metal lines, and AF45 glass as a dielectric due to its already proven good properties for $\mathrm{rf}$ signals $\left(\varepsilon_{\mathrm{r}}=6.2\right.$, $\tan \delta=9 \cdot 10^{-4}$ at $1 \mathrm{MHz}$ ) [5]. Figure 2 shows the dimensions chosen, where the thickness of the aluminum layers is $1 \mu \mathrm{m}$ $(\mathrm{t})$, the thickness of the AF45 wafer is $500 \mu \mathrm{m}(\mathrm{H})$, the width of the signal line is $100 \mu \mathrm{m}$ (W), the width of the grounded lines is $500 \mu \mathrm{m}(\mathrm{GW})$ and the distance between the signal line and the grounded lines is $25 \mu \mathrm{m}(\mathrm{G})$.

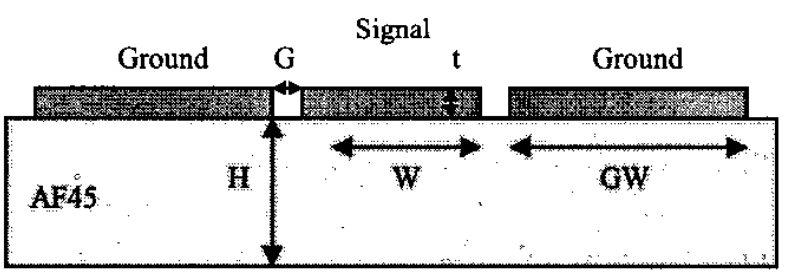

Figure 2. Cross section of CPW.

\section{Sensing capacitor}

The sensing capacitor is the moving part of the sensor, and, as it was explained in the operation principle section, we will estimate the power of the signal traveling through the CPW from the movement of such a capacitor. The design chosen for the moving capacitor was a double sided clamped metal membrane on top of the CPW as shown in figure 3, connected to ground with the grounded CPW lines. The membrane is completely made of aluminum and the dimensions are $300 \mu \mathrm{m}$ for the distance between the clamped sides (w), $1 \mu \mathrm{m}$ for the thickness of the membrane (h) and three different dimensions for the width (L): 100 , 1800 and $3600 \mu \mathrm{m}$ in order to study the response of the systems adding different capacitance values, which are: $49.2 \mathrm{fF}, 0.88 \mathrm{pF}$ and $1.77 \mathrm{pF}$ respectively. We decided to use a complete aluminum membrane instead of silicon or other materials with much better mechanical properties than aluminum mainly because when you add to such material the metal layer to act as a capacitor plate, effects like charge trapping and temperature dependence due to different expansion coefficient inside the membrane (which is added to the same effect between substrate and the membrane that always remains) are taking place. Because we have to measure very small displacements (over nm range), these effects could make the power detection impossible. In the other hand, due to the fact that only very small displacements are present on the sensing membrane, no yield is expected in the aluminum, and elastic response is always expected.

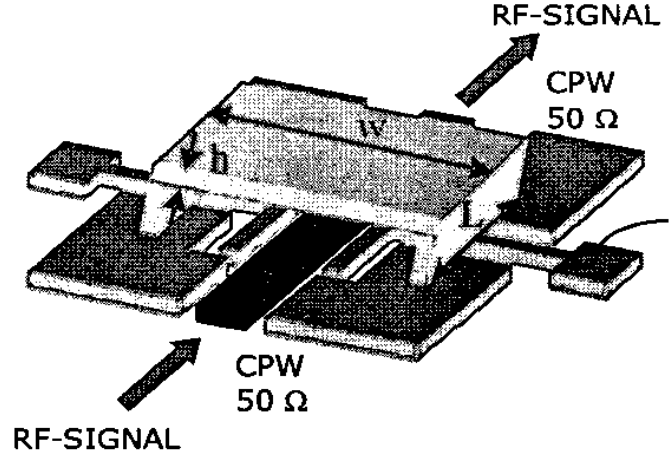

Figure 3. 3D draw of the sensor design.

The last step of the design was the placement of a sensing electrode which will be used to measure the displacement of the membrane. As we can see in figure 3, at a certain place, the ground lines let space for a floating metal layer below the membrane. Then, the displacement of the sensor is deduced from the difference in capacitance between this new layer and ground (where the membrane is connected).

\section{FABRICATION}

Figure 4 shows an overview of the fabrication process. After preparing the AF45 glass wafer with a cleaning process in nitric acid, the first step is the deposition of the aluminum metal layers needed for the CPW conducting lines (figure 4.a). The deposition was done by sputtering and the pattering of the layers by normal lithography process, in order to have the dimensions required and discussed in the design section ( $t, W, G$ and $G W$ on figure 2). The next step is the deposition of the sacrificial layer that will define the gap between the CPW and the moving plate that will be placed on top (figure 4.b). For this step, we chose as a material $1.7 \mu \mathrm{m}$ thick photoresist (Olin 907-17). In order to get rounded edges, a thermal reflow step was performed. After the patterning of the sacrificial layer, a layer of aluminum was deposited by low power sputtering ( $700 \mathrm{Watt})$ in order not to burn the photoresist and avoid stress (figure 4.c). The thickness of this aluminum layer is $1 \mu \mathrm{m}$. Finally, the membrane is released by oxygen plasma etching of the resist (figure 4.d), since any other wet etching technique would cause sticking problems of the membranes.

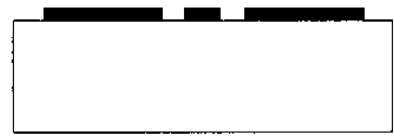

Figure 4.a

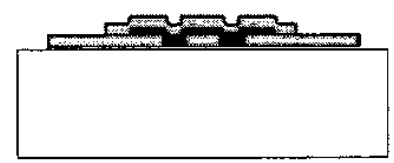

Figure 4.c

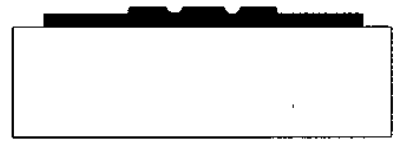

Figure 4.a

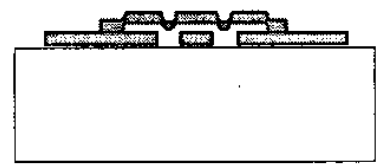

Figure 4.d
Figure 4. Schematic of the fabrication. 
SEM pictures of the final result in the fabrication are shown in figure 5 and 6.

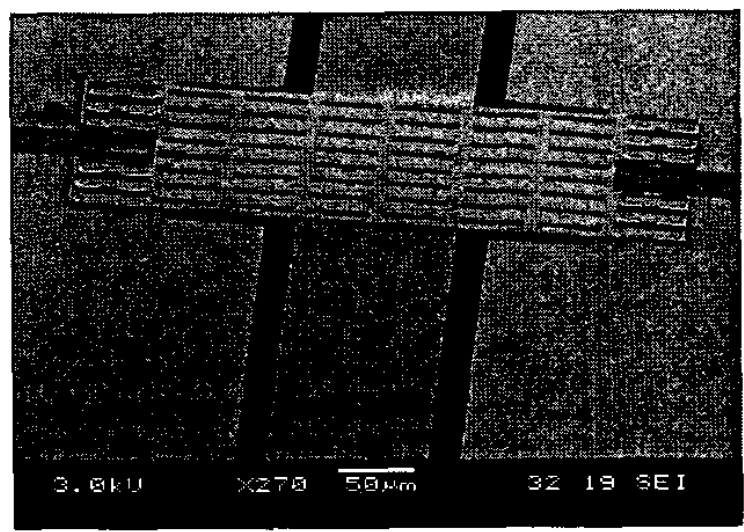

Figure 5. SEM picture of the sensor (top view).

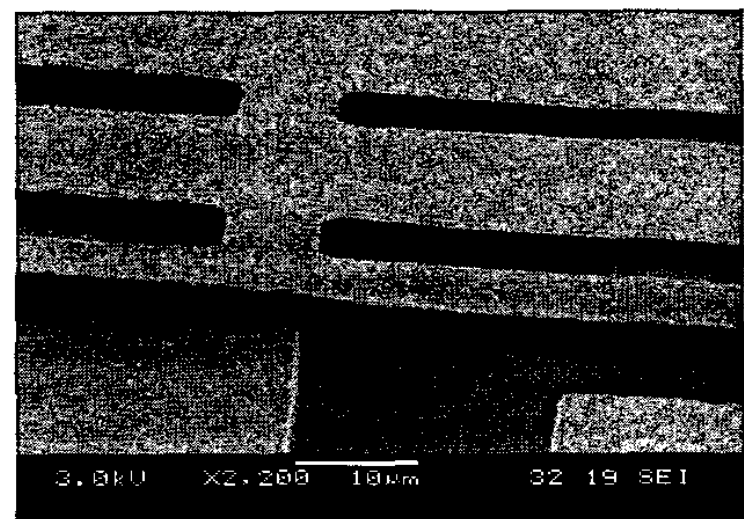

Figure 6. SEM picture of the sensor (close up).

\section{EXPERIMENT RESULTS}

Two kinds of experiments are needed for the characterization of the system. On one hand, we have to characterize the response of the system when rf signals are used. That means the study of reflection and transmission parameters of the signal that is applied to the sensor. On the other hand, we have to characterize the power measurements obtained with the sensor.

\section{S-parameters measurements}

The rf characterization consisted of the measurement of Sparameters with an HP $8510 \mathrm{C}$ Network Analyzer and using a Cascade Microtech 9000 probe station. Figure 7 shows $\mathrm{S}_{11}$ measurements compared with theoretical calculations from a CPW with different membrane dimensions.

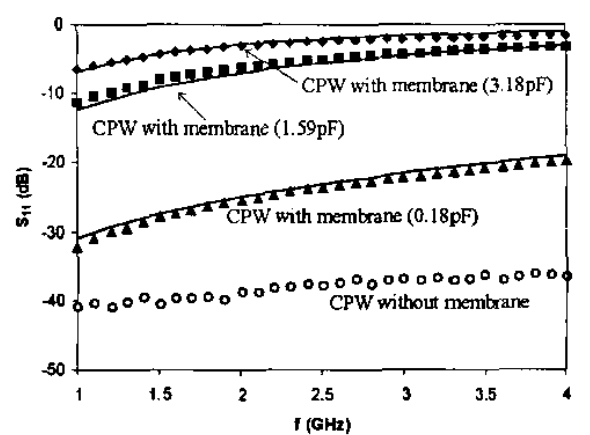

Figure 7. Theoretical (lines) and experimental (symbols) results of the $S_{11}$ parameter.

A good agreement between theory and experiments is presented, therefore, any $S_{11}$ response can be achieved (always above of the CPW limitation) by using the correct capacitance value. For the theory, the change in characteristic impedance due to the added capacitance is considered without fitting parameters.

Figure 8 shows $S_{12}$ measurements confronted with theoretical calculations from a CPW with the small membrane dimensions ( $100 \mu \mathrm{m}$ width), and measurements from the CPW without any capacitance. The transmission losses are in good agreement with the CPW losses presented in [6], where Ohmic losses of $0.04 \mathrm{~dB} / \mathrm{mm}$ are predicted at $5 \mathrm{GHz}$. Since the length of the CPW is $6 \mathrm{~mm}$ (much longer than needed for the sensor), better results could be achieved by using shorter transmission lines. This indicates that the transmission losses due to the CPW itself are more important that the reflection losses from the membrane.

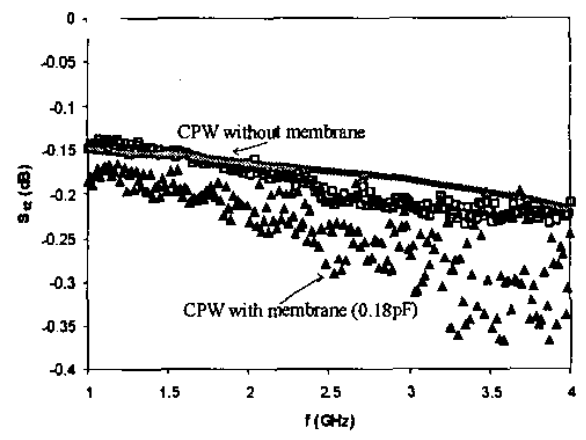

Figure 8. Theoretical (line) and experimental (symbols) results of the $S_{12}$ parameter.

\section{Power measurements}

The deflection of the membrane was detected by measuring the change in capacitance between the measuring electrodes and the ground using the electronics shown in the figure 9. 


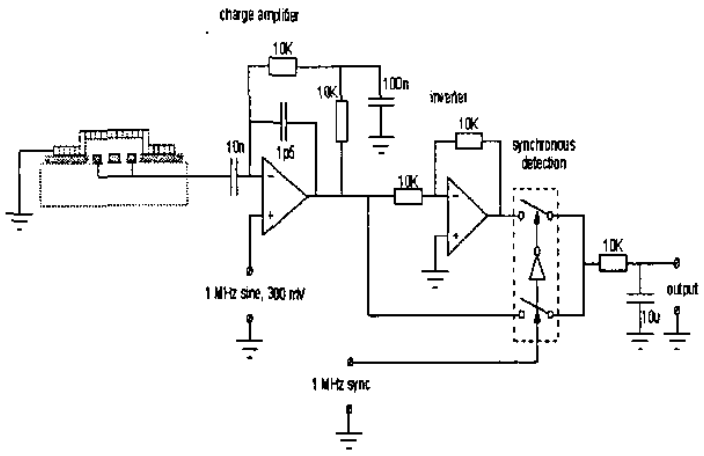

Figure 9. Schematic of the electronics used for capacitance read-out.

The dc voltage at the output is proportional to the capacitance between the membrane and the measuring electrode, and then the deflection of the membrane is detected.

The first movement measurements were done by applying a dc voltage, $V_{d c}$, between the central line and the ground of the CPW (and therefore, between the central line and the membrane). Figure 10 shows the measured quadratic relationship between the increase of $C$ and $V_{d c}$. The electrical force is proportional to $\mathrm{V}_{\mathrm{dc}}{ }^{2}$ and gives a linear displacement of the membrane.

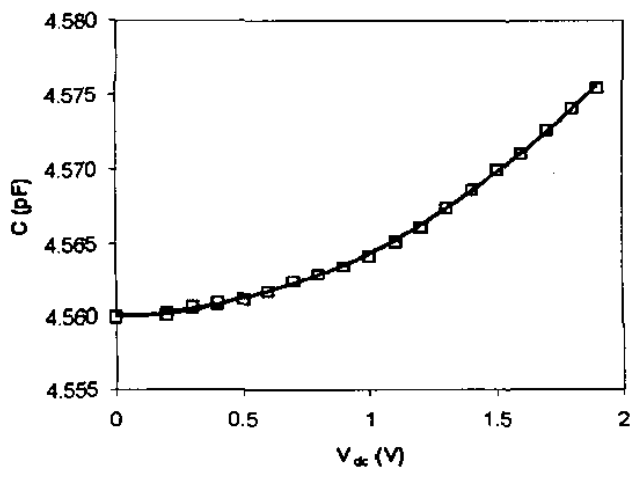

Figure 10. Sensor response applying dc voltage.

When we apply rf signals we are controlling the power, and a linear relation between capacitance change and if power is then expected. This is confirmed by the measurements in figure 11, obtained for signals of $2.5 \mathrm{GHz}$. From this graph we can easily deduce that the sensitivity factor of the hf power measurement is: $\mathrm{dC} / \mathrm{dW}=0.437 \mathrm{fF} / \mathrm{mW}$. From the dc measurements we have found a different sensitivity. The exact cause of this difference is currently being investigated.

\section{CONCLUSIONS}

A new power sensor for if signals with no dissipation during the power detection has been presented. First prototypes have been designed and fabricated. $\mathrm{S}$ parameters have been measured and compared with theoretical results showing a complete agreement between them. First power measurements for if signals at $2.5 \mathrm{GHz}$ have been performed proving the operation principle of the sensor. The sensor response at different signal frequencies and its linearity with the input power is now under further investigation. Discrepancies between the sensitivity for dc and rf signals will be studied in more detail.

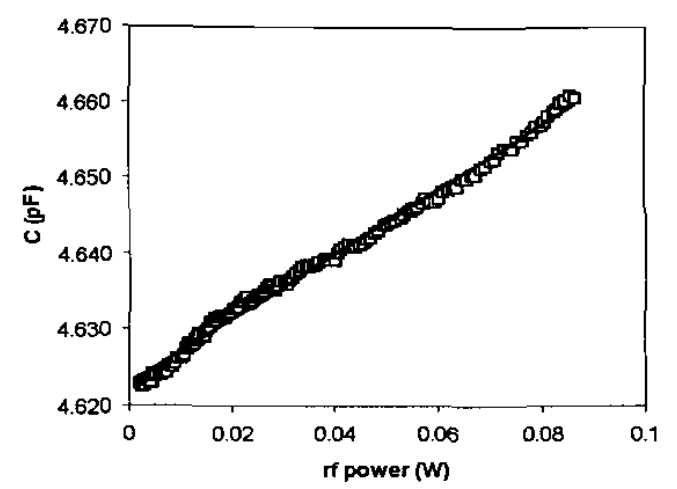

Figure 11. Sensor response applying if signal of 2.5 $\mathrm{GHz}$.

\section{ACKNOWLEDGEMENTS}

This work is part of the IST-2000-28261 EMMA program of the European Commission. We wish to thank Tauno Vähä-Heikkilä, Jukka Kyynäräinen, Aarne Oja, Jussi Varis, Heikki Seppä and Anu Kärkkäinen from VTT for useful discussions. Furthermore, we wish to thank Paul Roberts and Simon Hollingworth from Fluke for their help with the packaging study of the sensor.

\section{REFERENCES.}

[1] Seppä H., Kyynäräinen J., Oja A.: "Microelectromechanical systems in electrical metrology", IEEE Trans. Instrum. and Meas., Vol. 50 Issue: 2, pp 440-444, 2001.

[2] Tauno Vähä-Heikkilä, Jukka Kyynäräinen, Aame Oja, Jussi Varis, and Heikki Seppä, "Capacitive MEMS power sensor", MEMS WAVE Workshop, Crete, 2002.

[3] L.Fernández, J.Sesé, J.Flokstra, R. Wiegerink, "Capacitive MEMS application for high frequency power sensor", Micromechanics Europe, Sinaia, Rumania, October $6^{\text {th }}-8^{\text {th }}, 2002$.

[4] http://www.femlab.com

[5] http:/www.us.schott.com/tgd/english/products/af45.ht $\mathrm{ml}$

[6] H. Jansen, S. Pamidighantan, W. de Raedt, G. Beccarini, O. Dimonaco, D. Baert and H. Tilmans, "Micromachined devices for space telecom applications", pp. 87-94, $3^{\text {rd }}$ round table on Micro/NanoTechnologies for Space, ESTEC, Noordwijk, The Netherlands, May 15-17, 2000. 